\title{
On Applications of Self-Phased Array Antennas to Mobile Communications
}

\author{
Sergey L. Loyka and Vladimir I. Mordachev \\ Belorussian State University of Informatics \& Radioelectronics \\ P. Brovki Str. 6, Minsk 220027, Republic of Belarus \\ 375-(0)17-2398994, e-mail: loyka@nemc.belpak.minsk.by
}

\begin{abstract}
A simple method of substantial reduction (up to 1-2 orders) of power of base station and mobile unit transmitters used in mobile communication systems by the use of a self-phased array antenna which employs a pilot signal has been proposed. The increase in talk time and the reduction of battery weight by this method is also possible. Estimations of transmitted power reduction and examples of frequency assignment are given. Influence of electromagnetic environment on the self-phased array operation is discussed.
\end{abstract}

Key words: Self-phased array antenna, mobile communications

\section{Introduction}

Widespread utilisation of mobile communications systems which has lead to concentration of their transmitters over the limited territories (large populated and industrial areas) has dramatically aggravated electromagnetic and ecological compatibility problems. The radiated power of these systems' base stations can reach $5-10 \mathrm{~kW}$ (up to $320-350 \mathrm{~W}$ per channel), which can be ecologically dangerous in case their antennas are installed in residential districts as well as in the vicinity of industrial premises. Because of their comparatively large power, portable $(0.5-5 \mathrm{~W})$ and mobile $(5-50 \mathrm{~W})$ units represent significant danger to their users. [1-3]. Approximately half the radiated power of a hand-held mobile phone is absorbed in a person's head [4]. According to certain data, the radiated power level of portable units which is acceptable from the medical point of view is $100-300 \mathrm{~mW}$ [5]. Apart from the ecological danger, a lot of base and mobile units in a mobile communications system over the limited area impairs the electromagnetic environment to a substantial extent, which causes many EMC problems. Besides, power consumption and, consequently, battery weight and capacity are very acute problems in mobile units design [6].

\section{The Use of a Self-Phased Array Antenna}

The above-mentioned problems can be fully solved by substantial reduction of the power radiated by mobile communications system transmitters. In this case the service areas of these systems may retain their size if a transition to the microcellular level is made as well as if sensitivity of the receivers is increased or antennas gain is expanded. There can be no possible increase in sensitivity of receivers since this value is close to the limit (taking into consideration the state-of-art technological level and external noise level). It is hard to expand the gain of the mobile unit antenna because of the limitations on its weight and size. Transition to the microcellular level substantially increases cost and complexity of mobile communications systems and requires significant expansion of the radio frequency resource in order to ensure the intercellular communication.

The most promising way to retain the communication range of a system and size of its service and radio interference areas in this case is to use directional antennas for base stations so that the effective radiated power will be constant.. Due to the random direction of a signal received by the antenna from a mobile unit it is necessary to employ the selfphased array antenna, which automatically forms the main lobe in the direction of the received signal [7-9]. This antenna type facilitates not only signal reception from any direction by its main lobe, but also signal reradiation in the same direction [7]. The theory and technology of these antennas were developed earlier owing to the fact that they are used for satellite communications systems where the exact positioning of an antenna to the satellite is required. There are several different types of self-phased array antennas. We will take into consideration the self-phased array antenna which employs the pilot signal, because of its simplest design. The typical block diagram of this antenna is represented at Fig.1. It includes array elements $e_{l}-e_{N}$, self-focusing modules $u_{1}$ - $u_{N}$ and a summer $\Sigma$. The selffocusing modules include filters tuned to the pilot and primary (information) signal frequency, a pilot signal 


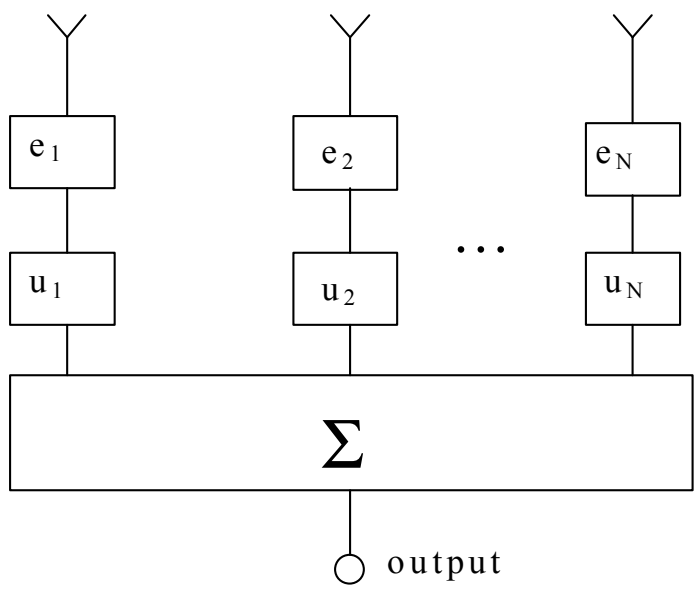

Figure 1. Block diagram of a self-phased array antenna. $e_{1}-e_{N}$ - are array elements; $u_{1}-u_{N}$ - are self-phasing units; $\Sigma$ - is an adder.

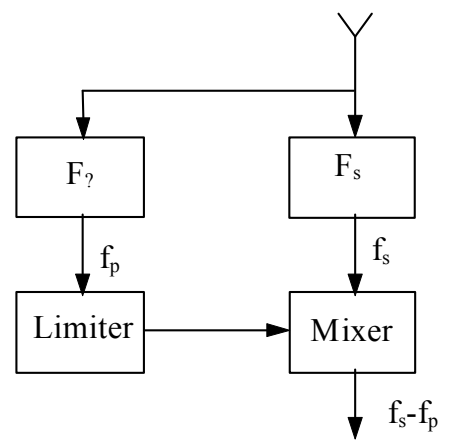

Figure 2. Block diagram of the self-focusing module. $F_{p}, F_{s}$ - are filters tuned to the pilot signal frequency $f_{p}$ and the primary signal frequency $f_{s}$.

limiter, and a mixer (Fig.2). The filter tuned to the intermediate frequency is connected after the summer. Phase shift compensation is carried out in the mixer during the multiplication of the pilot and primary signals. The signal phase at the intermediate frequency is identical at the output of all elements. In this case the main lobe direction always coincides with the direction of pilot signal reception. The operating principles of these antennas are described in detail in [7-9].

Let us estimate the advantage gained due to the reduction of power radiated by the base station transmitter, which can be achieved by using the selfphased array antenna. The constant character of a communication range (radius of a base station service area) determines the constant character of the effective radiated power

$$
P_{e}=G \cdot P_{t}=\text { const }
$$

where $P_{t}$ - is a transmitter power, $G$ - is an antenna gain.

Hence it follows that in case the antenna is a nondirectional one in the horizontal plane, for example, the dipole, the required transmitter power is

$$
P_{t, d}=\frac{P_{e}}{G_{d}}=\frac{P_{e}}{1.64}
$$

where $P_{t, d}$ - is the transmitter power for the dipole, $G_{d}$ is the gain of this dipole. The advantage due to the reduction of power radiated by the transmitter (admissible power reduction) is

$$
K=\frac{P_{t, d}}{P_{t}}=\frac{G}{1.64}
$$

where $P_{t}$ - is the transmitter power for the self-phased array antenna; $\mathrm{G}$ - is the gain of this array. If the selfphased array is designed as a circular array antenna which consists of dipoles then, taking into account the shadowing effect, $G \approx 1.64 \cdot N$, where $N$ - is the number of phase array elements (for the conversion factor in selffocusing modules equal to unity). Then the ratio (3) takes the form

$$
K \approx N
$$

Thus the use of the self-phased array antenna for the base station facilitates reduction of power radiated by its transmitter and a mobile unit transmitter by $10-20 \mathrm{~dB}$ (for $N=10 \div 100$ ). In this event the required transmitter power value for the cellular communications system will not exceed 0.01-0.1 W, and for the mobile unit paging system with active response - 0.05-0.1 W, which means that the most stringent sanitary requirements for the mobile communications system are satisfied without increasing the number of base stations. Additional advantages of using the self-phased array antenna are as follows:

- The opportunity of increasing the continuous talk time of mobile units by 1-2 orders while preserving the capacity of batteries which encourages the further development of the radio paging with active response;

- The opportunity of decreasing the battery weight.

- The directional properties of the self-phased array antenna used for the base station make it possible to considerably reduce the volume of the radio frequency resource actually used by the station (size of the radio-interference zone determined using probability-statistical criteria). 
Further, we would like to note a number of possible limitations and peculiarities connected with using the self-phased array antenna for the mobile communications system:

- In the case of multichannel system structure it is necessary to use a pilot signal which has a different frequency for each channel. In this event the pilot signals of separate channels can be distributed either within a separate bandwidth, or within the guard band between the neighbouring channels (Fig.3).

?) $\quad$ p - pilot signal; s - primary signal.
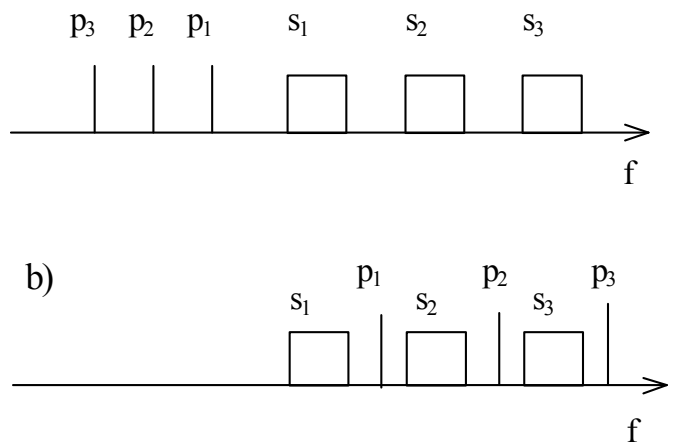

Figure 3. Frequency distribution of primary and pilot signals.

- The pilot signal is a harmonic one, and it is possible to carry out its filtering in the self-focusing module by the narrowband filter whose pass band can be by 2-4 orders less than the pass band of the filter for the primary signal and can be chosen taking into account the danger of dynamic effects in the system. Hence, on tentative estimations, the pilot signal power can be by $20-40 \mathrm{~dB}$ less than the primary signal power, which is very important for ensuring intrasystem EMC of base stations and mobile units which comprise the mobile communications system.

- The use of the self-phased array antenna will raise the cost of base stations and mobile units as well as the corresponding operational costs. However, on tentative estimations, this raise will be rather small.

- The use of adaptive phase array antennas for mobile communications systems [17-19] is complicated owing to the sharp deterioration of their characteristics when the number of mobile units which work simultaneously is close to the number of adaptive phased array elements, complexity of the adaptation procedure and substantial amount of adaptation time, and complexity of an adaptive array design.

\section{Influence of Electromagnetic Environment on Self-} Phased Array Operation

Electromagnetic environment can essentially influence the self-phased array operation due to the active elements which are present in its structure. This influence can manifest itself in two main ways: (1) distortion of the array pattern due to multipath propagation (reflections) or jamming signals, and (2) generation of the intermodulation products at the array output ("intermodulation lobes").

Presence of reflections (multipath propagation) or jamming (interference) signals at the pilot signal frequency can lead to significant deterioration of a selfphased array operation. Both the pilot signal channel and the primary (information) signal channel can substantially influence on the self-phased array operation and must be taken into account during the analysis [10-12]. In the case of the threshold amplitude characteristic of the pilot-signal channel, directivity of self-phased array under conditions of multipath propagation can be written as follows [10-12]:

$$
G=G_{p} \cdot G_{s} \cdot G_{0}
$$

where $G_{0}$ - is the array directivity in the absence of reflections; $G_{p}$ - is reduction in directivity due to cutting off some channels (influence of reflections by way of the pilot signal channel); $G_{s}$ - is reduction in directivity owing to variations in amplitude and phase of the primary signal (influence of reflections by way of the primary signal channel). As the detailed analysis shows, the average reduction in directivity under normal operation conditions is no more than $6 \mathrm{~dB}$, but more larger reduction is possible in some specific cases.

Presence of intermodulation lobes in a self-phased array can substantially decrease its interference immunity (or, equivalently, increase its susceptibility to interference) and distort output signal. It should be noted that the array pattern at an intermodulation frequency may be quite different from that at the working frequency A detailed analysis of intermodulation lobes in active array antennas can be found in [13-16].

\section{Conclusion}

A self-phased array antenna is very promising for the use in mobile communications. Its design is very simple: it performs self-phasing without phase shifters and adaptive control electronics. The large progress in the field of microwave IC technology makes its production very simple and cheap.

By and large, the use of the self-phased array antennas in mobile communications systems requires for 
the development of a special standard which would regulate the use of radio frequency resource and the ensuring of EMC for base stations and mobile units.

\section{References}

[1] I. Shtrikman, and H. Fruchting, 'Numerical Determination of the Performance and of the Electromagnetic Compatibility of Antennas for Hand Held Mobile Telephones', Proceedings of 13th Wroclaw Int. Symp. on EMC, pp.189-193, Wroclaw, Poland, 1996.

[2] M. Fischetti, 'The cellular phone scare', IEEE Spectrum, pp.43-47, June 1993.

[3] H.J. Mikolajczyk, and M. Kamedula, 'Direct Experimental Evidence of Microwave Influence on the Central Organs of Endocrine System', Proceedings of 13th Wroclaw Int. Symp. on EMC, pp.130-132, Wroclaw, Poland, 1996.

[4] Y. Rahmat-Samii and K.W. Kim, Antennas and Human in Personal Communications: Applications of Modern EM Computation Techniques, $12^{\text {th }}$ Int. Confer. on Microwaves and Radar (MIKON'98), Krakow, Poland, May 20-22, 1998, pp.36-55.

[5] Sanitary norms for admissible levels of the physical factors concerning the use of the consumer goods in household conditions. - Sanitary regulations 9-29-95 (Russian Federation. 2.1.8.042 -96).

[6] D.C. Cox, 'Wireless Personal Communications: A perspective'. In "The Mobile Communications Handbook", Editor-in-Chief J.V. Gibson, CRC Press, 1996.

[7] P.V. Brennan,: 'An Experimental and Theoretical Study of Self-Phased Arrays in Mobile Satellite Communications', IEEE Trans. on Antennas and Propag., Vol.37, No.11, pp. 1370-1376, 1989.

[8] M.J. Withers et al 'Self-focusing receiving array', Proc. IEE, Vol. 112, No.9, pp.1683-1688, Sep 1965

[9] 'Antenna arrays: methods of calculation and designing. A survey of foreign sources', Ed. by L.S. Benenson, Moscow, Sovietskoe radio, 1966.
[10] S.L. Loyka, Influence of Pilot Signal on Directivity of Self-Phased Arrays Under Conditions of Multipath Propagation, IEEE Trans. on EMC, vol.40, No.1, pp.12-18, 1998.

[11] S.L. Loyka, On Operation of Self-Phased Arrays Under Conditions of Multipath Propagation, Int. Conf. on Microwave \& Radar (MIKON-98), Poland, Krakow, 1998, pp.535-539.

[12] Loyka S.L. 'Directivity of Self-Phased Arrays in Conditions of Multipath Propagation,' Proceedings of the Seventh International Conference on HF Radio Systems and Techniques, East Midlands Conference Centre, Nottingham, UK, 7-10 July, 1997.

[13] S.L. Loyka, Characteristics of receiving intermodulation channel of active array antennas, International Journal of Electronics, Vol.80, No. 4, pp. 595-602, 1996.

[14] S.L. Loyka, Interference Immunity of Active Array Antennas in Rigid Electromagnetic Environment, Proceeding of the XI International Microwave Conference "MIKON-96", Poland, Warsaw, 1996, vol.1, pp.57-61.

[15] S.L. Loyka, Intermodulation receiving channels in active array antennas, Izvestia Vuzov. Radioelectronica (Radioelectronics and Communications Systems, Allerton Press Inc.), vol.39, N 2, 1996, pp.68-74.

[16] S.L. Loyka, Conditions of existence of main lobes in two-signal spatial selectivity characteristic of active array antennas, Izvestia Vuzov. Raioelectronica (Radioelectronics and Communications Systems, Allerton Press Inc.), vol.39, N 6, 1996, pp.3-9.

[17] R.A. Monzingo and T.W. Miller, Introduction to Adaptive Arrays'. John Wiley \& Sons, New York, 1980.

[18] B. Widrow and S.D. Stearns, 'Adaptive Signal Processing'. Prentice-Hall, Inc., Englewood Cliffs, 1985.

[19] D. Nowicki, J. Ronmeliotis, 'Smart Antenna Strategies', Mobile Communication International, April 1995, pp. 53-56. 